\title{
Classifying the Diversity of Bus Mapping Systems
}

\author{
Mohd Shahmy Mohd Said, ${ }^{\mathrm{a}, \mathrm{b}}$ and David Forrest ${ }^{\mathrm{a}}$ \\ ${ }^{a}$ School of Geographical and Earth Sciences, University of Glasgow, United Kingdom \\ ${ }^{b}$ Department of Surveying Sciences and Geomatics, Mara University of Technology, Malaysia
}

\begin{abstract}
This study represents the first stage of an investigation into understanding the nature of different approaches to mapping bus routes and bus network, and how they may best be applied in different public transport situations. In many cities, bus services represent an important facet of easing traffic congestion and reducing pollution. However, with the entrenched car culture in many countries, persuading people to change their mode of transport is a major challenge. To promote this modal shift, people need to know what services are available and where (and when) they go. Bus service maps provide an invaluable element of providing suitable public transport information, but are often overlooked by transport planners, and are under-researched by cartographers. The method here consists of the creation of a map evaluation form and performing assessment of published bus networks maps. The analyses were completed by a combination of quantitative and qualitative data analysis of various aspects of cartographic design and classification. This paper focuses on the resulting classification, which is illustrated by a series of examples. This classification will facilitate more in depth investigations into the details of cartographic design for such maps and help direct areas for user evaluation.
\end{abstract}

Keywords: Cartographic Design, Bus Maps, Transit Mapping, Public Transport Maps

\section{Introduction}

Bus maps play an important role in portraying bus routes and other information for public transport users. Users may depend on the map to make their decision during their travel planning process and as well as during travel. With the current rapid development of cities around the world and advancement of bus transportation systems, the task of properly formulating and presenting adequate of information in just a single map may not be an easy task. It is important to understand the current cartographic representation variations used in bus maps. With an understanding of the variation, the map makers will have a better perspective on delivering the right information in the most appropriate style.

The process starts with examining a wide range of bus maps from around United Kingdom, European countries and the United States of America. There is no limitation on the maps' published date as the authors also seek to find the variation in bus map design over the time. The publishing dates of the maps included in this study range from 1975 to 2017. All sample maps were evaluated using an objective evaluation form. The data collected from this form were the basis of multiple statistical analyses to identify the current pattern and technique used by map makers.

The first level of distinction that has been identified is between geographically correct bus maps and schematic bus maps. The geographically correct map approach can be identified as map that uses information and design of a topographic map or street map as the basis and background. The bus routes are drawn to follow the original shape of natural and man-made features on the base map without any significant distortion and change of line shape. These geographically correct maps have been the most popular choice in designing bus map in the past especially before the year 2000. In contrast, schematic maps can be characterised as a map that depicts the bus route in various straight line forms, typically either in horizontal, vertical or at 45 degrees declination (Morrison 1996). Well known and clearly effective for metro systems, there is a view that schematic maps as defined are not suitable for use for bus mapping (Morrison 1996), but the number of bus maps that adopt this approach has risen significantly in recent times. More than half of bus map in this sample are some form of schematic, with the majority published after the year 2000 .

\section{Use of Geographical approach to represent bus route}

Although the schematic approach is gaining popularity in new bus map design, the geographical approach is still an important approach to portray bus routes. There are certain differences in how cartographers represents the bus route using this geographical approach. The most popular style is one that directly takes all the main features of a topographic map and puts all this information as background information on a bus network map. This style is known as the 'Full Road Network'. This style identifies the bus route or bus services by highlighting the route using distinctive colour. The colour selected for routes always has high contrast with the colour(s) used for the background. The service numbers typically appear above the road. This style is exemplified by a transportation map from Amsterdam (Figure 1).

The 'Main Road Only' style is another approach used in Geographical bus maps. As defined in its name, here only main roads are shown on the map. Other major features, 
such as water bodies, open spaces and rail lines, may also be included in the map, but not the full road network. To help users identified the bus services lines, the bus services numbers are placed alongside the roads being used as the route lines. An example can be found in a transport map of Glasgow (Figure 2).

A third style in this geographical approach is the 'Bus route only'. This style looks similar to the 'Main Road Only' style, but in this style, the bus routes are the only features that are highlighted with a strong colour. The text, numbers and labels are only placed on the bus service's road. Background information may vary from being very subservient to non-existent.

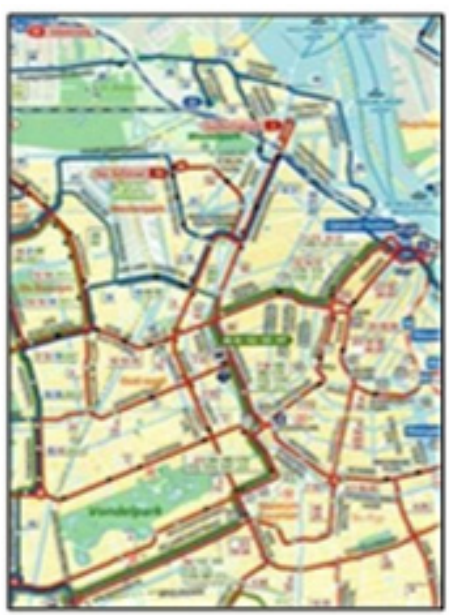

Fig. 1. Full network bus map (Source: Amsterdam Lijnennetkaart 2012)

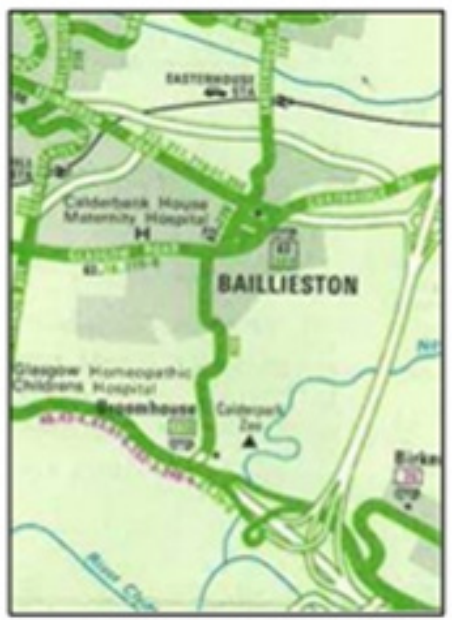

Fig. 2. Main Road Only bus Map (Source: Greater Glasgow Transport map 1975)

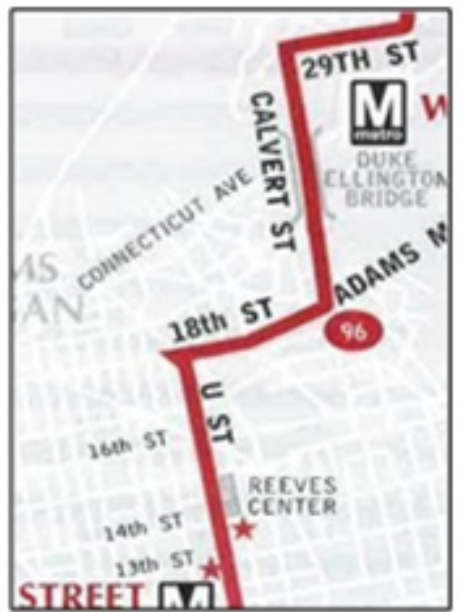

Fig. 3. Bus Route Only bus map (Source: 96,97 Metrobus 2015)

\subsection{Methods used to differentiate bus services}

In a bus network map, there will be various individual bus route services and sometimes, multiple transportation modes in addition to buses. - To differentiate modes and routes; in the geographical approaches' cartographers commonly use colour, number (text), line style or combination of these.

\subsubsection{Differentiation from other transportation modes}

The first method to differentiate the modes is by using colour. In case of the bus map that shares with the other transportation modes (such as trains and trams), the cartographers often use colour as the feature to single out the bus services. The large range of possible colours makes this option the easiest choice for cartographer to create a distinctive representation for each transportation mode. For example, on Figure 4, the cartographer uses two different colours (red and blue) to distinguish between Tram service routes and Bus service routes.

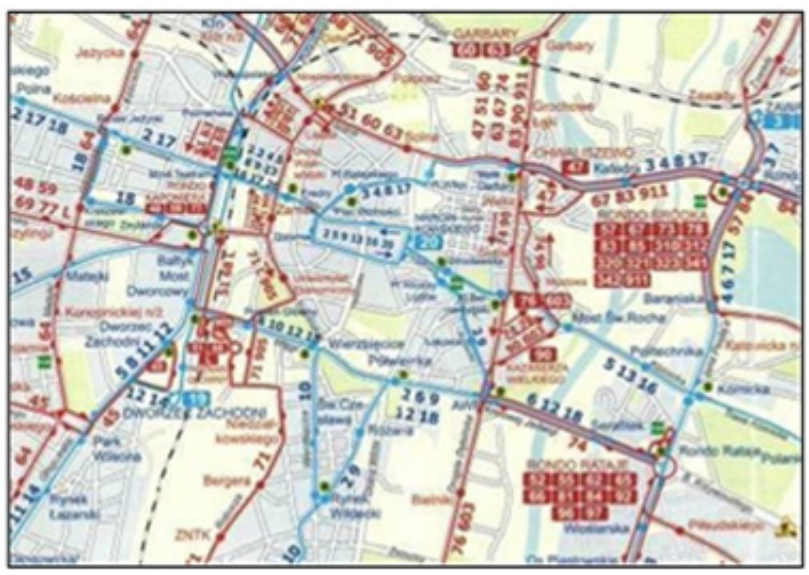

Fig. 4. Use of Colour for Transport's Mode Differentiation (Source: Map Network Connections ZTM Poznan 2014)

The other option to differentiate the bus routes from the other transport modes is by using both combinations of colour and line style. This combination gives more options for the map maker to differentiate the modes. As we can see from the Figure 5, the train mode is represent in green colour while the bus route is represent in the 
yellow- colour. The distinctive line styles of each mode make the difference very clear to the user.

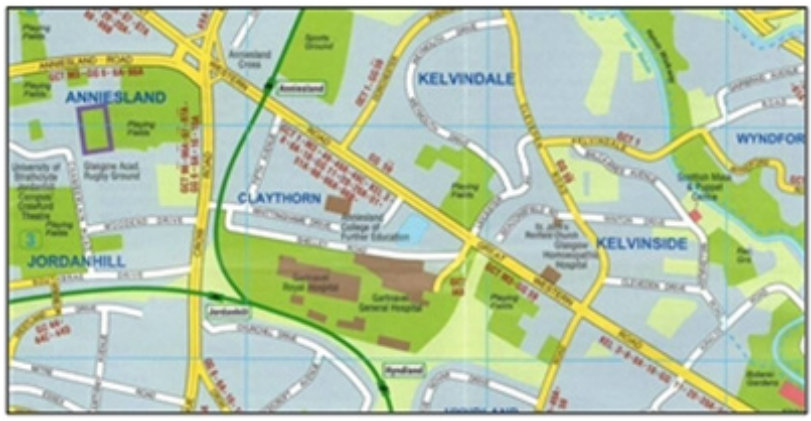

Fig. 5. Use of colour for Transport Mode Differentiation (Source: Map network Connections ZTM Poznan 2014)

\subsubsection{Differentiation between bus services}

Colour continues to be the most commonly used feature to distinguish between bus services. One example of the usage of colour to distinguish bus services can be found in a map from Washington (Figure 6). Here, four colours (green, yellow, red and blue) are used to individualize four different bus services. Colour is the only feature that changes and aspects; such as line shape; remained the same.

The second method used by cartographers is number or text. As the number of bus services shown increases, variations in colour or style become insufficient. Prior to 2000 , the usage of service number to differentiate the bus services was widely used. As we can see from the Oxford Bus Company map (Figure 7), the service lines are shown with the same colour throughout the map. The only way for the user to knows their service line is through the service numbers above the lines, which have the same colour as the bus routes.

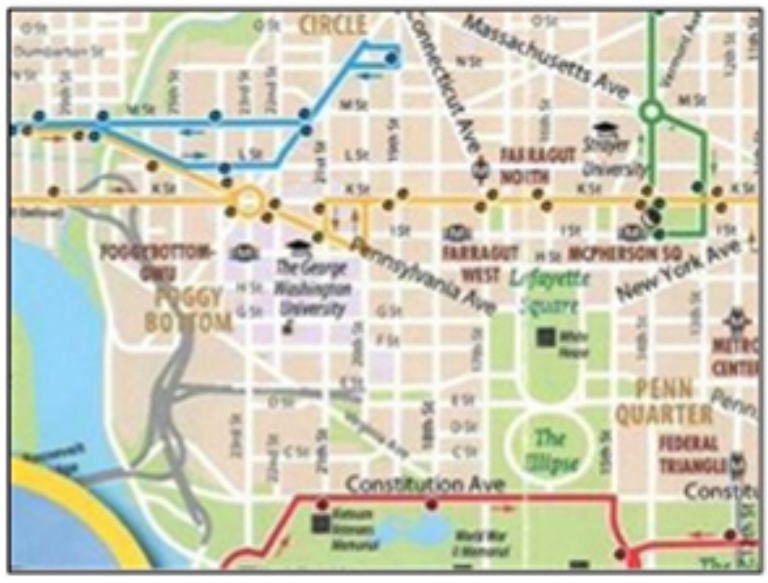

Fig. 6. Differentiation in colour for routes (Source: DC Circulator and Information Guide 2015)

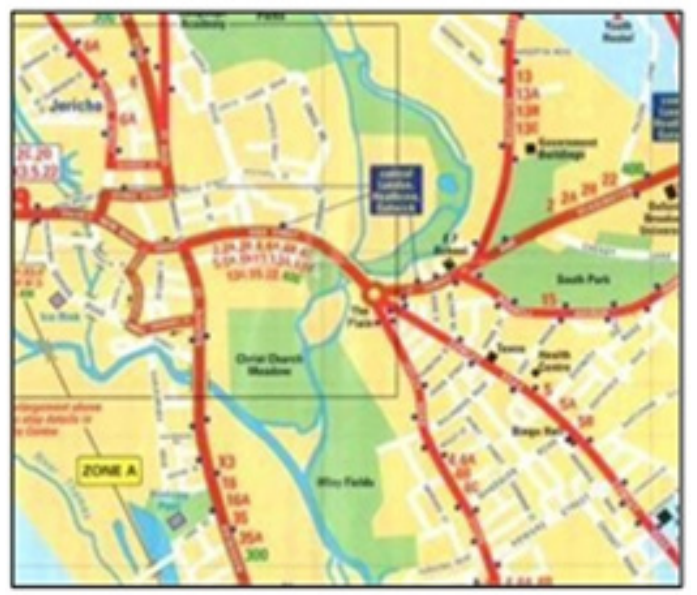

Fig. 7. Differentiation of routes using text (Source: The Oxford Express 1995)

Another method practiced in geographical maps is to combine the route number and route colour. In this case, the distinctive aspect can be found in the colour of the bus service's number. Every road being used as the bus service route has bus service numbers and the numbers are coloured based on the respective bus service. A bus map from London (Figure 8) is one example that shows how this combination of features works.

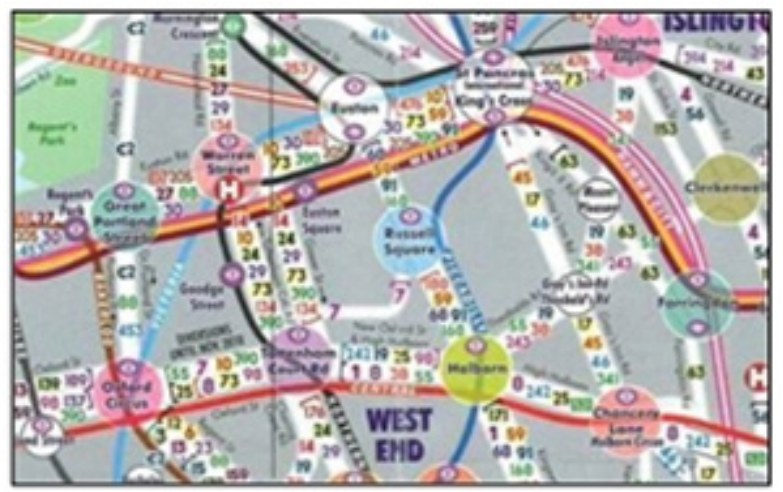

Fig. 8. Differentiation using combination of colour and text (Source: London by Bus 2011)

\subsection{Level of background information in the Geographical type of bus map}

To help users put the bus services in context, additional background information needs to be included in the map. The level of background information needs to be carefully considered so it is not too much, confuses the user, or make it difficult to find their selected bus line; But it should not be too little or the user will not know how to orientating themselves when using the map. In this study, we examine the representation of some main background features in bus maps.

Grids are almost always seen in national topographic maps. From the authors' observation, grids are not widely used a bus maps. A grid is only really useful if it is used to index information on the map, such as tabulating the start and end points of routes, or an index to other useful features. A map that uses grid as part of the background information is the bus map from Geneva (Figure 9). This map is very large. The grid can help the user to remember 
and find any particular location on the map after multiple uses.

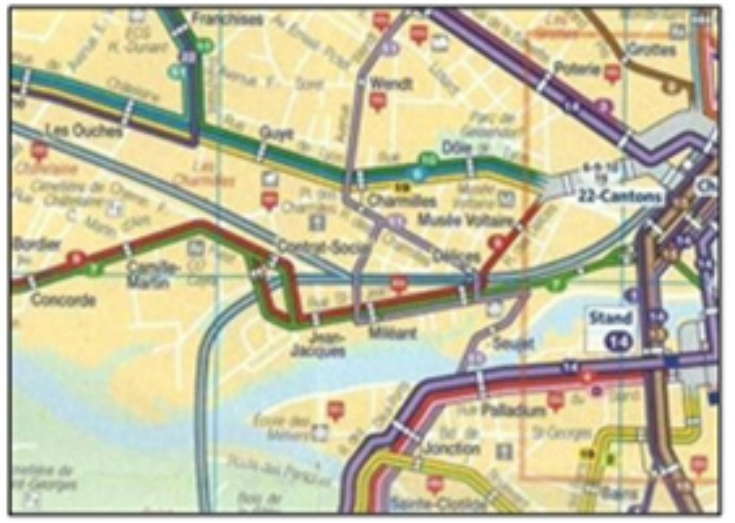

Fig. 9. Grid representation on bus map (Source: Geneve Plan De Reseau 2012)

A large bus maps may consist of various coloured routes, multiple line styles and many numbers to differentiate routes. These features appear against a background which will affect their visibility and legibility. It is important for bus map to have a good visual hierarchy with a good contrast between route and background images. The bus services line should be at the top of this hierarchy to highlight the main purpose of the map. An example of a geographical approach bus map with good hierarchical levels can be seen in Figure 10. This map uses a multicoloured background but we can still clearly see the hierarchical level between the bus routes and other features on that map. Even though one of the colours used to define bus services is similar to that for the river and these features overlap at some points, the bus service line can still be identified clearly.

However, not all bus maps have successfully portrayed the visual hierarchy. For example an Edinburgh map (Figure 11), predominantly uses variations in colour lightness to show both routes and background which does not provide sufficient contrast. This could lead to confusion to user while using this bus map.

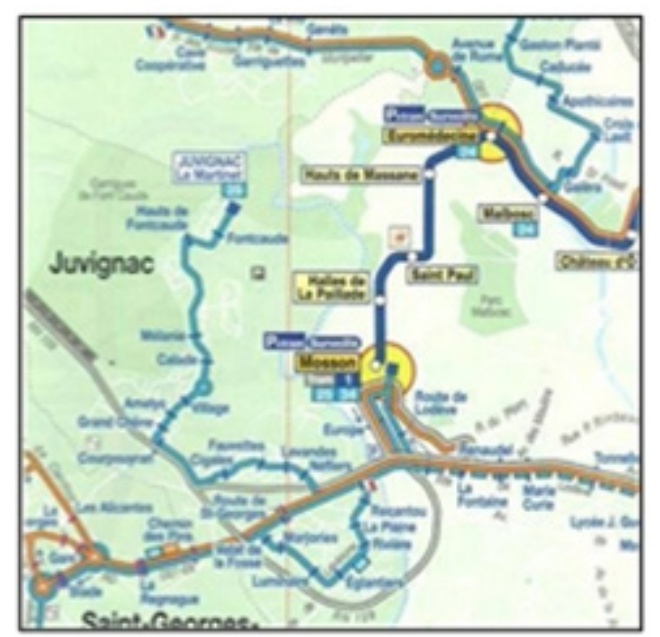

Fig. 10. Good hierarchy level and background image (Source: Montellier Plan De Reseau 2010)

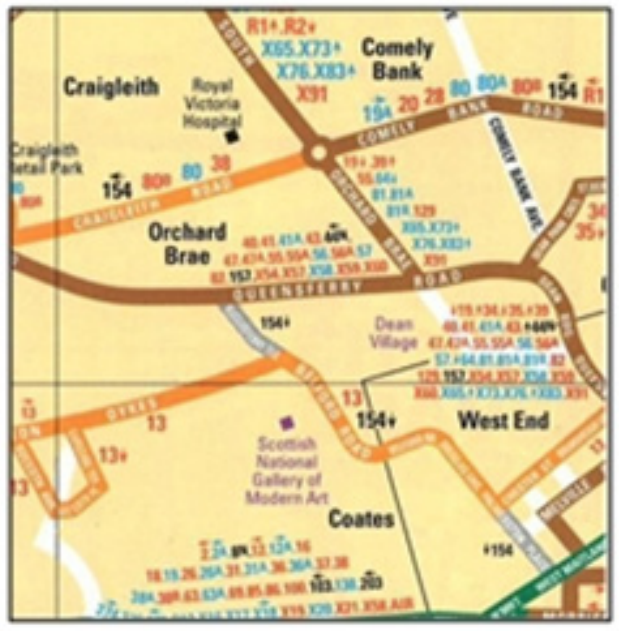

Fig. 11. Bad hierarchy level and background image (Source: Edinburgh Travel Map 2010)

\section{Use of Schematized representation to portray bus transportation system}

A simple, comprehensive definition of 'schematic map' is difficult to determine. According to Oke \& Siddiqui (2015), a schematic map is a linear cartogram of a given network. Schematic diagrams use symbolic representations of pathways to reduce complexity and ease orientation in a network. This is the definition of schematic map used in this paper. The use of schematic maps in transportation is frequently based on the design of London Underground Map by Harry Beck in the 1930s, which some cartographer view as a masterpiece of map design (Guo 2011). The use of schematized representation in bus maps can be divided into two major styles, the first is a fully schematic design (typically used to map metro and rail systems) and the second is the semi-schematic design. In general, fully schematised representations retain topology (connections) but vary scale to such an extent that geography becomes highly distorted. On the other hand, the semi-schematic design merges elements of the schematic map to locally simplify the representation, but retains an overall geographicallycorrect approach. Thus the semi-schematic design is a compromise between a fully schematic map and truly realistic geographical representation.

From the maps studied, it is found that when cartographer uses the schematized representation, they tend to use the fully schematized approach rather than semi-schematized approach. The surge in the usage of both kind of schematized representation for bus maps can be been seen to start from around the year 2010. This maybe tied in with availability of software to generate such representations. An example of a fully-schematic design style is shown in Figure 12 and an example for a semischematic design is shown in Figure 13. 


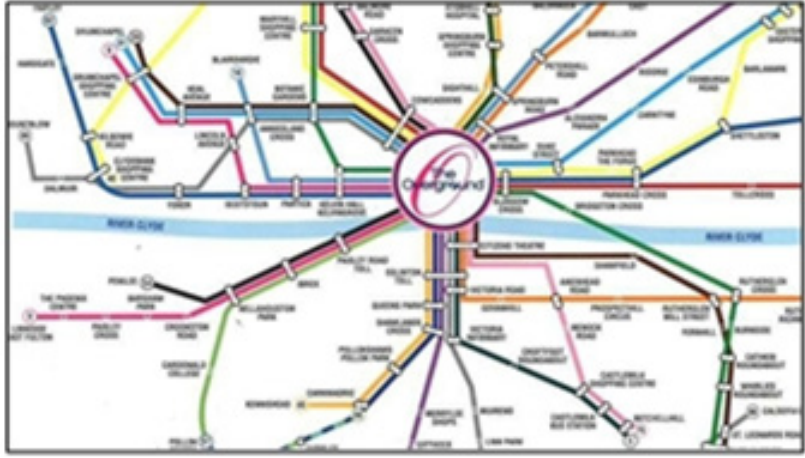

Fig. 12. Fully- Schematic Bus Map (Source: Overground Glasgow First Bus Map 2012)

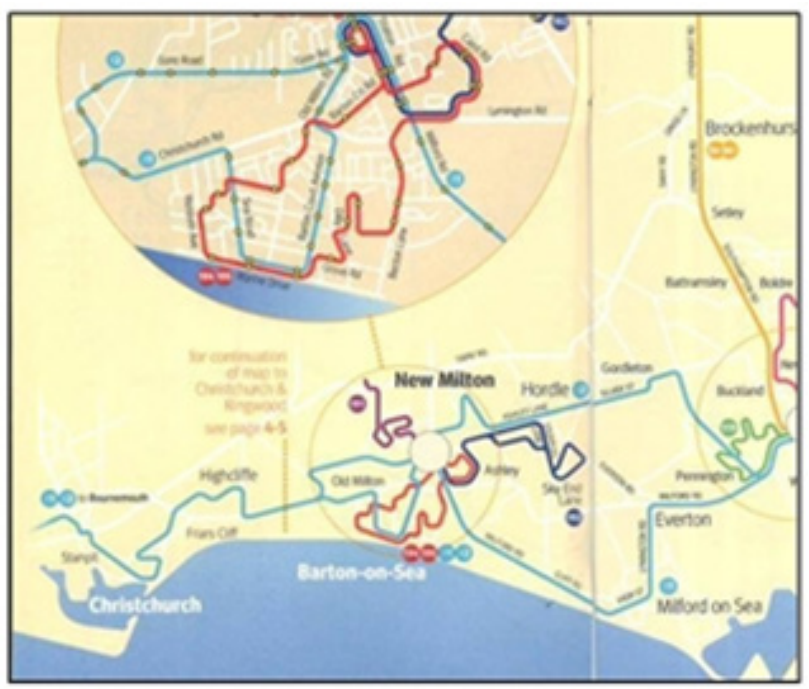

Fig. 13. Semi - Schematic Bus Map (Source: Bus Guide - New Forrest, Christchurch, New Milton, Ringwood 2010)

\subsection{Method use to differentiate bus services}

\subsubsection{Differentiation from Other Transportation Modes}

There four types of design features used to differentiate modes of transportation in a schematized representation. The features are the differentiation using colour, number, line shape or combination of two of these. Combination of methods is widely used as it gives a lot of options for cartographers to provide a distinctive appearance to several transportation modes. As we can see from the bus map from Chicago (Figure 14), the difference between train mode and bus mode is made using both line style and colour. The trains have a different colour used to represent their routes (various strong colours) and use a thick line, while the bus routes use a low contrast colour with a small line width. There is also a map that differentiates the transportation mode using difference in colour only. As we can see from the Virginia Metro Bus System map (figure 15), the train mode and the bus mode are differentiated using colour. The least used feature to differentiate transportation modes in schematized map is line style. In the Ile de France transport map (Figure 16), both the train and bus systems are represent by the same colour, which is red, the only distinctive appearance between both of these modes is the line style (thicker and narrower).

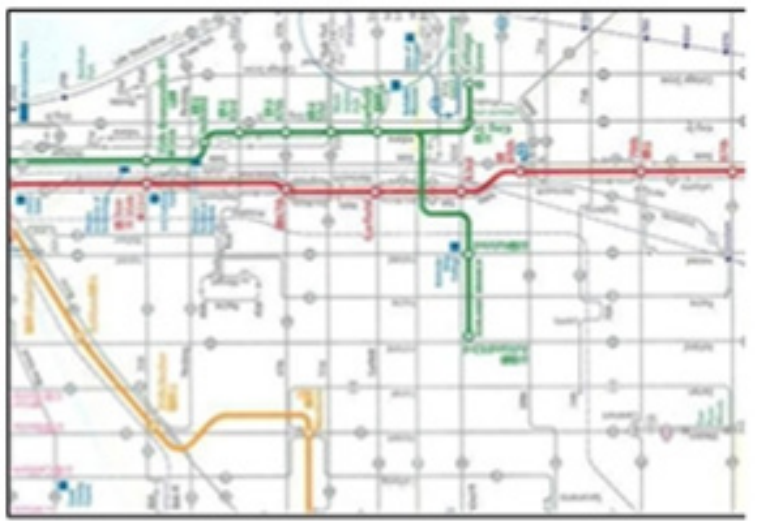

Fig. 14. Differentiation using combination of method in Schematic bus map (Source: Bus and Rail Map, Chicago Transport Authority 2010)

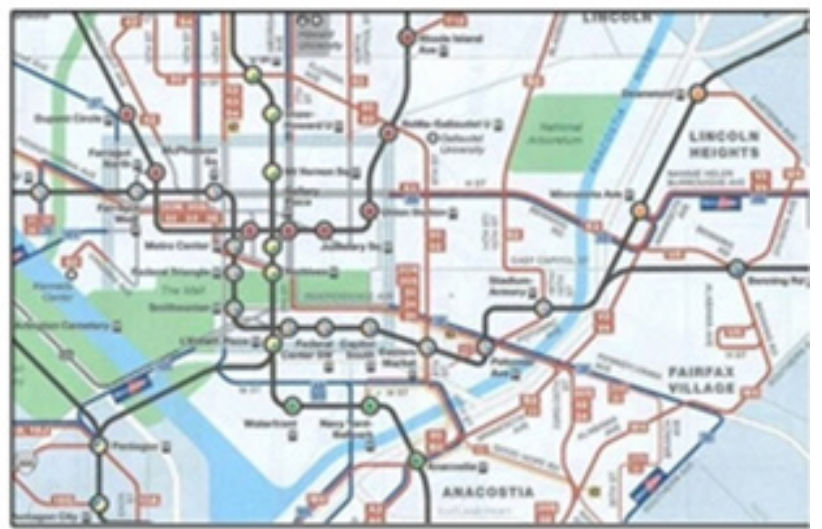

Fig. 15. Differentiation using colour in Schematic bus map (Source: Metrobus System Map - Virginia 2015)

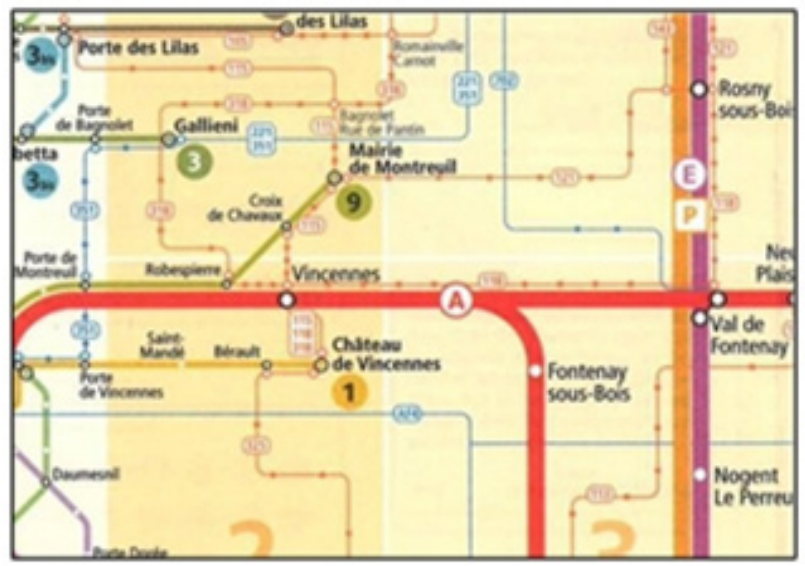

Fig.16. Differentiation using line style in a Schematic bus map (Source: Les transports en Ile-de-France 2011)

\subsubsection{Differentiation between bus services}

The most common method used to differentiate bus services in a schematized bus map is colour. This is different from previous discussed approaches, where combinations of two or three attributes are widely practised. As we can see from the bus map from France (Figure 17), a wide range of colours are used to represent bus services. In this case a different colour is used for each service. 


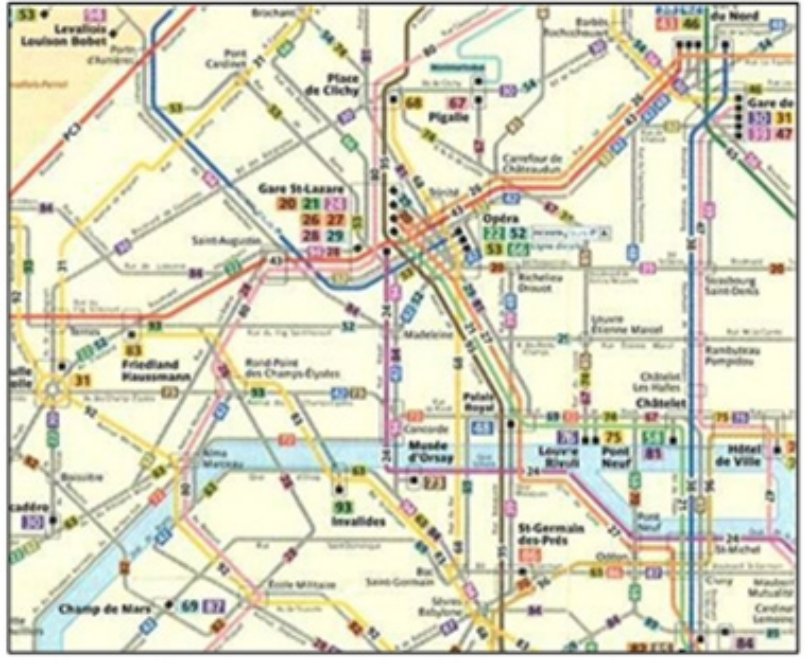

Fig. 17. Use of colour to differentiate bus services line (Plan de Poche - Paris, 2005)

The number (text) feature is the second most used method to differentiate bus services in schematized bus maps. Figure 18 show that the bus services are represent by the same colour which is yellow. In order to know the respective bus services, users need to read the number above the lines.

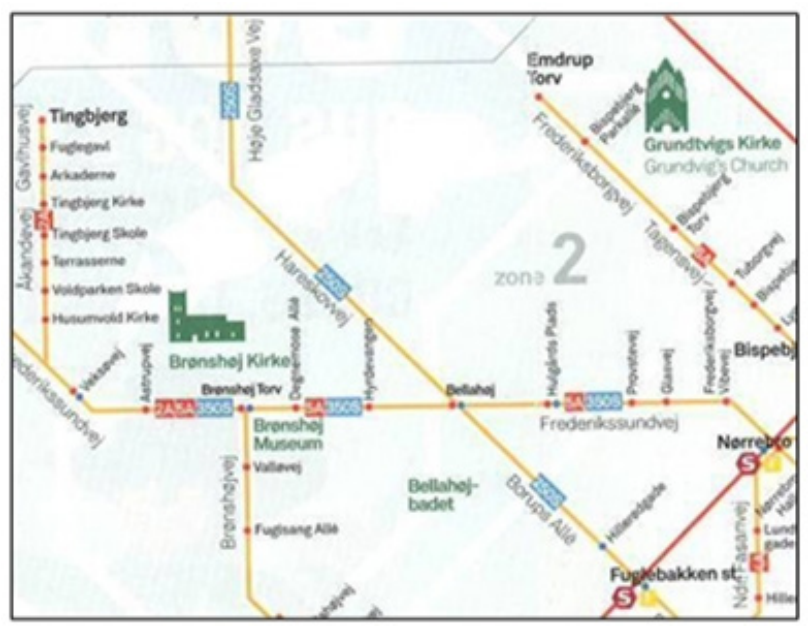

Fig.18. Use of text to differentiate bus services line (Plan de Poche - Paris, 2005)

\subsection{Level of Background information in the Schematic type of bus map}

Like geographical maps, to help users have a good understanding of schematic maps, additional background information may need to be included. The level of background information needs to be carefully considered so it does not hinder the user finding their selected bus line, or seem too little so that the user does not know how to orientating themselves during the usage of the map. There is the added complication in schematics maps of distorted geography, which can make the shape of geographical features unrecognisable.

Grids are not extensively used in schematized bus maps. As schematized maps already have a high degree of distortion during the schematized process, adding a grid may not give any help in determining locations.
There is stark contrast between the appearance of hierarchies in fully schematic style and semi-schematic styles. As semi-schematic maps may adopt the design and style use in geographically correct maps, the presence of hierarchical levels can be seen in all semi-schematic maps examined. This situation can be seen in Figure 19. In this map, the bus service lines are shown with strong contrasting colours. The colours look very different to the background. Despite the multi-colour background, the user can still see the bus service lines clearly and the background colours help them when using the map. This indicates a good hierarchical level of representation. The network of main roads also being included as background information further helps the user in orientating themselves.

However this is not the same with the fully schematized style. Fully schematic maps mainly focus on the route only. As shown in the Dresden Bus map (figure 20), there is no background information other than major water bodies. Users need to refer to other maps that contain the road network or other topographic features to help them in locating the exact position of the bus route.

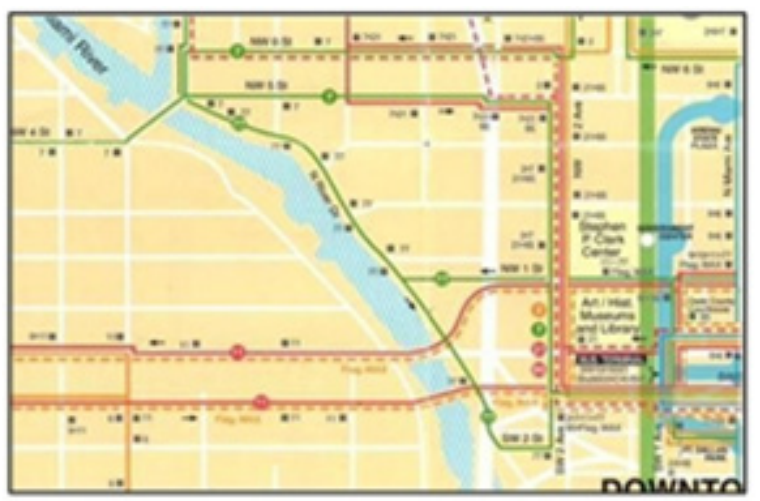

Fig. 19. Good hierarchical level in a Schematize bus map (Miami-Dade County Transit Map - 1996)

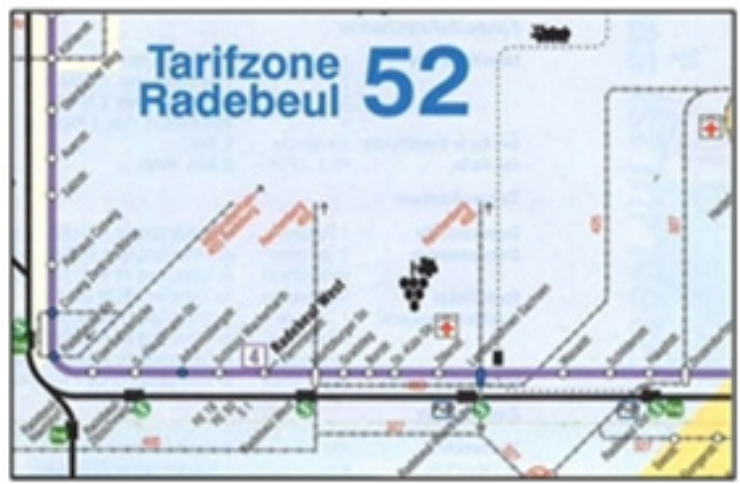

Fig. 20. Bad hierarchical level in a Schematize bus map (Dresdner Linien 2008)

\section{Conclusion}

Analysing and classifying in detail the current cartographic approaches to bus map design is indeed a challenging process. This is mainly because there are a variety of different cartographic design and visualization techniques that are currently being practiced. Nevertheless, the resulting classification developed by 
this study will help to identify the particular considerations that should be taken into account when designing a bus map in a specific context. Investigations of landmark incorporation in bus maps is a further features being investigated. Landmarks can be described as functioning reference points based on distinctive environmental feature (Vinson 1999). The area of investigation includes on the number of landmarks that should be included, the kind of landmarks that are most effective and the preferable methods for landmark symbolisation. A comprehensive report will be created based on this additional analyses. Furthermore, an ongoing research will use all these findings as a basis to develop an understanding of cultural preferences for approaches to bus map design.

\section{Acknowledgements}

The authors acknowledge that this paper uses examples from twenty public transport maps from all around the world. A big thanks to all map providers for the public transportation system in all of these cities - Amsterdam, Glasgow, Washington, Poznan, Edinburgh, London, Genève, Paris, Virginia, Miami-Dade and Dresden. Many other maps were consulted in developing the classification.

We acknowledge the support of the Malaysian Government and Mara University of Technology for providing the first author with a $\mathrm{PhD}$ Scholarship which has enabled this study.

\section{References}

Guo, Z., 2011. Mind the Map! The Impact of Transit Maps on Travel Decisions in Public Transit. Transportation Research Part a-Policy and Practice, 2011(45), pp.1-25.

Morrison, A., 1996. Public Transport Maps in Western European Cities. Cartographic Journal, The, 33(2), pp.93-110.

Oke, O. \& Siddiqui, S., 2015. Computers \& Operations Research Ef fi cient automated schematic map drawing using multiobjective mixed integer programming. Computers and Operation Research, 61, pp.1-17.

Vinson, N.G., 1999. Design Guidelines for Landmarks to Support Navigation in Virtual Environments. Proceedings of the Conference on Human Factors in Computing Systems (ACM SIGCHI), pp.278-85. 